\title{
Retinal detachment treated with intravitreal air: an evaluation of 241 cases
}

\author{
HECTOR B. CHAWLA AND JOSEPH A. COLEIRO \\ From Princess Alexandra Eye Pavilion, Royal Infirmary, Edinburgh
}

SUMMARY 241 unselected cases of retinal detachment treated since 1971 with cryopexy and intravitreal air are discussed. The series is analysed according to the position of the retinal tear(s), and we describe briefly the surgical technique and evaluate the main complications.

The unchanging aim of retinal surgery is to reattach a living retina with as little damage to it and the rest of the eye as is consistent with success-at a first operation. There are many methods of achieving this end, and all retinal surgeons have been forced by the demands of different cases to use most of them at one time or another. It is only when the surgeon is left with a choice that any controversy occurs.

\section{Methods}

The use of intravitreal air has already been described in detail (Chawla and Birchall, 1973; Chawla, 1974). The basic operation may be briefly summarised here. Monitored freezing is applied to the retinal break(s); subretinal fluid is released through a scratch sclerotomy over a carefully selected site; sterile air is injected into the vitreous through the pars plana; a fine silicone band (No. 40) is placed around the equator of the eye; the patient is then positioned postoperatively to float the break(s) uppermost against the frozen area(s) for at least 48 hours. Two variations from the original technique should be mentioned.

SUBRETINAL FLUID

Before piercing the choroidal knuckle we now first coagulate it with a round ball diathermy electrode in the manner advocated by Freeman and Schepens (1974) though without any transillumination.

LOWER TEAR(s)

All except large equatorial breaks, lying between the 5 and 7 o'clock meridians, are treatable with intra-

Address for reprints: Dr H. B. Chawla, Royal Infirmary, Princess Alexandra Eye Pavilion, Chalmers Street, Edinburgh EH3 9HA vitreal air. To raise the lower pole of the eye uppermost the patient is nursed head downwards in a tilted bed with a stitch around the inferior rectus muscle for 24 to 48 hours (see Fig. 1). Two main considerations determine whether patients are suitable for this method of treatment: (1) It must be possible to float the tear(s) uppermost; (2) the eye must be softened enough to make room for at least $1 \mathrm{ml}$ of intravitreal air. This is usually done by subretinal fluid release, hence we prefer balloon to shallow detachments, but it may be necessary to alter the volume with intravenous mannitol solution or in rarer cases by release of fluid vitreous along a Graefe blade through the pars plana (Fig. 2).

\section{AUGMENTED OPERATION}

Where tears are present in opposite quadrants, not treatable with a single air bubble, we have sealed the lower tear with a radial sponge implant on the equator or with a circumferential segment of guttered silicone band (No. 20) for peripheral break(s). Local counter-traction is sometimes needed in the presence of a rigid retina, either as a primary or as a secondary procedure.

Although Rosengren (1938) had already established vitreal air injection as a reputable method, we were reluctant at first to use it freely. However, its effective simplicity, speed, and freedom from complications have now persuaded us to give it a rapidly increasing share (now $90 \%$ ) of the retinal detachments treated in our service. Because of this earlier hesitation, the 241 cases discussed in this paper form less than $50 \%$ of our total numbers since 1971, but with 200 detachments per year to treat, it is now our procedure of choice. In this present group of patients the shortest follow-up is 6 months at the time of writing. 


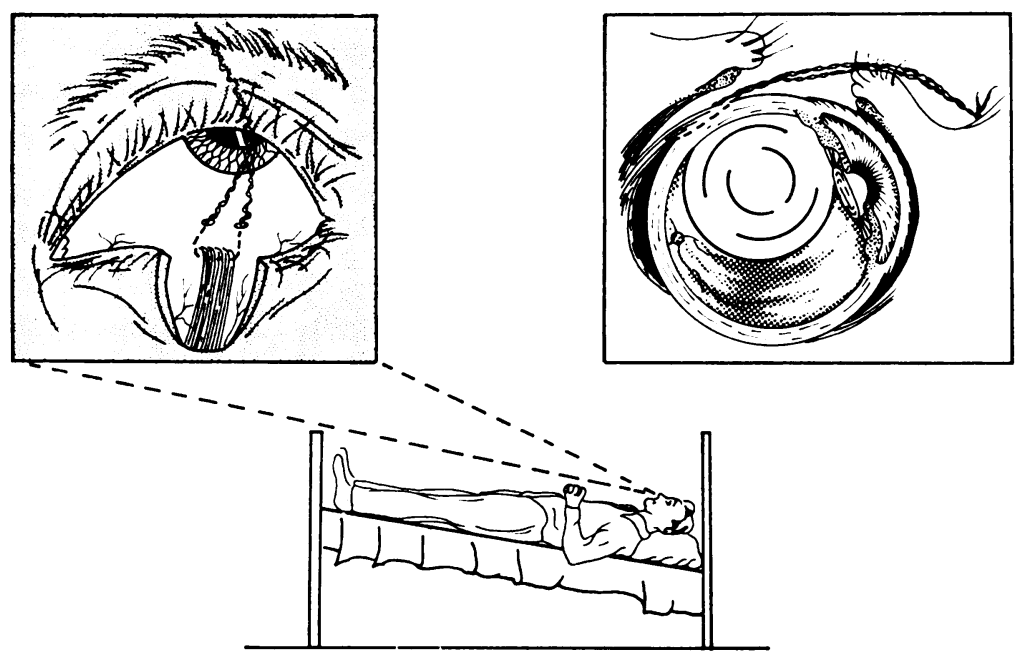

Fig. 1 Inferior rectus stitch. Tilted bed

We have broken the cases up according to the position and nature of the retinal tear(s), taking a flat retina as our main criterion of success but not dismissing the influence that the preoperative state of the macula and vitreous has had on central visual recovery (Chisholm et al., 1975).

EQUATORIAL BREAKS

Total: 155 .

Retina flat after:

(a) Primary basic operation: first attempt, 95; second attempt after (i) first operation, 33; (ii) other procedures, 14.

(b) Augmented operation: first attempt, 11; second attempt after (i) first basic operation, 1; (ii) other procedures, 1 .

Successes: $145(94 \%)$; failures: 10.

\section{FAILURES}

Total: 10.

Nine of these started with little hope of success from the outset and all ended with a shrunken retina. The tenth, a balloon detachment with a solitary horseshoe tear, developed a violent vitreal inflammation 3 days after surgery. Although this subsided on treatment with high systemic doses of steroids and antibiotics, the vitreous cleared only enough to reveal a rigid, partially detached retina.

Of the others 4 might have reasonably been rejected for surgery anyway on the grounds of fibrosis. In another eye a giant horseshoe tear could be just seen through a vitreous haemorrhage. Another, also with a presenting vitreous haemorrhage, had already had argon laser photocoagulation to curb hypertensive new-vessel formation. In 2 cases no healthy sclera could be found after sponges were removed from areas of widespread diathermy which had already induced similar destructive effects in the retinae. The last, grossly myopic and staphylomatous, eye required the easy removal of fluid vitreous because of a shallow detachment. This eye

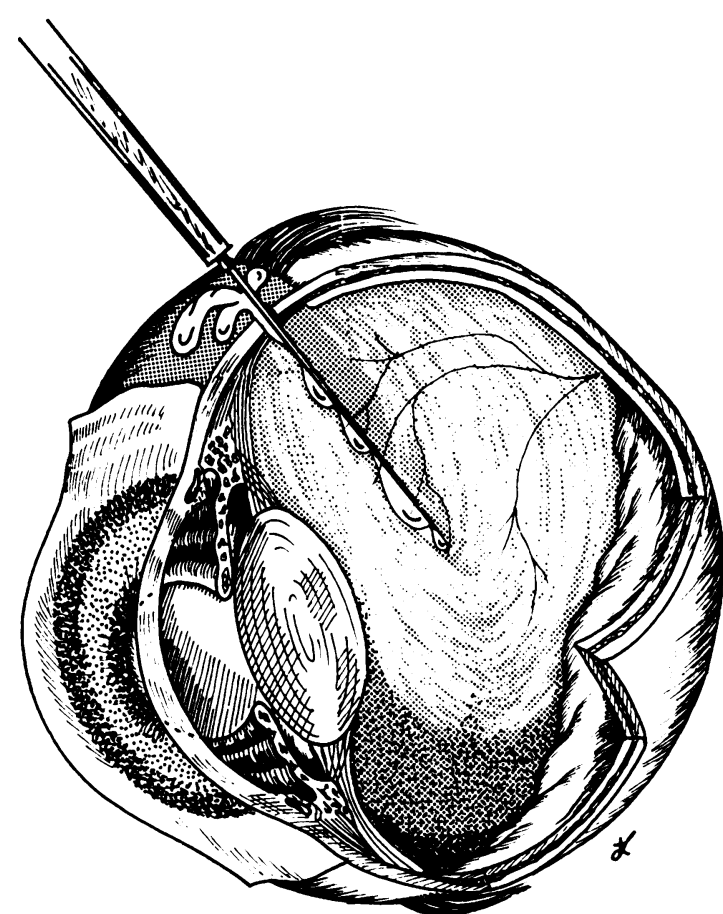

Fig. 2 Release of fluid from within the vitreous cavity 
never recovered from the shock of surgery and collapsed quickly into phthisis.

\section{APHAKIC DETACHMENTS}

Total: 49.

(1) Genuine aphakic tears.

Retina flat after

(a) Primary basic operation: first attempt, 15; second attempt after (i) basic operation, 1; (ii) other procedures, 1 .

(b) Augmented operation: first attempt, 1; second attempt after (i) basic operation; (ii) other procedures, 1 .

(2) Other tears with incidental aphakia.

Retina flat after

(a) Primary basic operation: first attempt, 11; second attempt after (i) basic operation; (ii) other procedures, 4.

(b) Augmented operation: first attempt, 1; second attempt after (i) basic operation, 1; (ii) other procedures.

Successes: 36 (73\%); failures: 13 .

\section{FAILURES}

Total: 13.

Aphakic detachments, even the straightforward ones, have a rather sinister reputation that we have not been able to confirm. All but 1 of our failed cases started out with a poor prognosis but in only 4 of these could gross vitreous loss at cataract extraction be held to blame. Of the remaining 9 there was 1 in which vitreous blood partially obscured the retina. The next very myopic eye became phthisical; 2 more presented with advanced fibrosis.

Three had suffered previous surgery. The first, a young man, lost the lens after a penetrating injury which tore the retina along the equator with a fair degree of vitreal haemorrhage. The primary augmented operation held this retina flat for about 6 months before shrinkage took it beyond the scope of a second surgical attempt. The second, also in the same age group, had had multiple needlings for congenital cataract. A basic operation flattened the retina for a year, after which he relapsed with a fresh tear and suffered a vitreous haemorrhage at the second operation. This was thought to be due to the cryopexy. The third was virtually inoperable following excessive diathermy.

The next eye, on top of its other problems, had a successful corneal graft for interstitial keratitis. Two basic operations failed because a posterior polar tear was not seen at first. This retina flattened partially after a third basic operation during which the cryopexy caused a choroidal haemorrhage; that the graft has survived all these surgical insults may reflect well on the gentleness of the technique.
DIALYSIS

Total: 24.

Spontaneous including one giant tear.

Retina flat after

(a) Primary basic operation: first attempt, 18.

(b) Augmented operation; first attempt, 1.

Traumatic.

Retina flat after

(a) Primary basic operation: first attempt, 2; second attempt, 1.

Successes: $22(91 \cdot 6 \%)$; failures: 2 .

\section{FAILURES}

Total: 2 .

The first, a moderate myope, with 3 small temporal dialyses, became a surgical catastrophe. A posterior sclerotomy was performed to reduce the intraocular pressure, which had risen out of control for no detectable reason during the operation, and he suffered a vitreous haemorrhage. The second with traumatic retinal detachment and lens subluxation and vitreous haemorrhage, flattened for 6 months before relapsing with preretinal retraction.

\section{MACULAR AND POSTERIOR POLAR HOLES}

Total: 13.

Retina flat after

(a) Primary basic operation: first attempt, 6. One of these suffered three days postoperatively an acute inflammatory episode in the vitreous which cleared on high doses of steroid and antibiotics. Second attempt after (i) basic operation, 1; (ii) other procedures, 3.

Successes: 10 ; failures: 3 .

Because the retinal elevation in post-polar detachments does not usually advance beyond the equator, subretinal fluid has to be let out beside the more posterior and hence larger choroidal vessels. Should this prove impossible, then, to make way for the air bubble, fluid vitreous can be released in much the same manner as that described for posterior sclerotomy in malignant glaucoma (Scott and Smith, 1961).

\section{FAILURES}

Total: 3 .

All of these cases were highly myopic. In 2 no explanation could be offered for the failure except poor uptake of cryopexy at the posterior pole. The third eye collapsed into intractable hypotony.

\section{COMPLICATIONS}

In our other cases, as well as in those of this series, the most common cause of choroidal or choroidovitreal haemorrhage is cryopexy, despite careful monitoring of the iceball. However, disabling 
vitreous haemorrhage occurred in just 2 cases, and flat choroidal haemorrhage gravitated to the macular area in only 3 instances.

Subretinal fluid release produced no significant bleeding but did produce incarceration of the retina on 6 occasions ( 2 in one patient with bilateral shallow detachments and multiple holes). Because the release site was chosen with this complication in mind, all of these were contained with ease and safety with a little extra cryopexy and the same air bubble or a small scleral infolding buckle.

Choroidal detachment masquerading to the unwary as an actual retinal detachment becomes a complication only when large enough to affect vision. A deliberately eccentric encircling band which blocked half the vortex veins was the culprit in 2 cases which settled when the band was cut. Two further patients complained of a deep ocular discomfort refractory to routine analgesics. The bands were sectioned and the pain settled, but neither these nor any of the others in our series showed any signs of anterior segment ischaemia.

Vitreous inflammation occurred only twice. Of these cases 1 cleared to a great extent, though leaving large trailing vitreous floaters. The second cleared only enough to reveal a traction detachment.

In 1 case a blunt needle allowed air to strip the pars plana. Although in this particular eye the retina was already in the grip of fibrosis, this complication is not irreversible and the air can be removed, just as one would remove subretinal fluid, quite easily, provided its presence in the wrong place is recognised. This mishap can be eliminated by watching for the needle tip before depressing the plunger.

\section{Discussion}

It is the current orthodoxy to disfavour the use of intravitreal air for 5 main reasons. It is alleged that the air bubble cannot remain long enough to be useful; that air can bring about permanent changes in the vitreous; that the release of subretinal fluid is too dangerous a practice; that the air bubble can introduce infection; that one cannot visualise the retina through the bubble. This is not our experience.

From the first attempt's success rate alone there can be no reasonable doubt that the air bubble does in fact remain long enough to carry out its task. In none of our cases (even those 8 with diabetes) did the vitreous develop any adverse features that might fairly be ascribed to the harmful effect of air.

Subretinal fluid release need not be dangerous if one does not take liberties with the basic rules. The release site must be scrupulously chosen and marked where the retinal detachment is deep, where the major choroidal vessels can be easily avoided, and where an accidental retinal perforation or incarceration can be contained either by the air bubble or by a small scleral infold. In practice the ideal point lies over the centre of a major equatorial tear should one such be available. If fluid vitreous apnears, as it may rarely do, in place of subretinal fluid, the eye is still softened and thus satisfactorily prepared for the intravitreal air injection.

We have found only 1 choroidal-vitreous haemorrhage that could have resulted from this manoeuvre, and, contrary to present belief, we would blame cryopexy as the commonest cause of such haemorrhages, which, if present, occurred prior to the release of subretinal fluid. As for intravitreal infection, only 2 eyes developed a posterior vitreal inflammation, and only 1 of these was left with permanent disability as a result.

If the air is introduced from a dry syringe with a single movement of the plunger, then the bubble will also be single and the retinal view as informative as it was before the injection, provided one views the fundus with the binocular indirect ophthalmoscope, which is unaffected by the gross change in refraction of the eye.

The release of fluid vitreous along a Graefe blade is always a last choice in detachments with multiple holes where just too much subretinal fluid exists to allow tear sealing with a buckle but not enough to allow its safe release. Preoperative slit-lamp examination of the vitreous is essential in all cases, for although deep detachments always present with a very fluid vitreous, so frequently may shallow detachments, which progress slowly as a result of either tiny tears or of a partially solid vitreous cortex. A shallow retinal detachment with a relatively 'healthy' vitreous is most likely to settle with local buckling or encirclement to catch the posterior tear edge, because the retina in these cases is detaching reluctantly.

The inferior rectus stitch has extended the use of air to all inferior tears except those on the 6 o'clock equator, which lie usually too far posterior to be brought uppermost by positioning. Any discomfort due to the stitch is lost in the general discomfort of the operation. The head-down position, although less than pleasant, is maintained rigorously for only 24 hours, and no stitch is ever allowed to remain beyond 48 hours. There has been no evidence of postoperative impairment of extraocular muscle action.

The apparent emphasis on the discussion of failures aims to demonstrate that many of these cases were given the chance of an operation without any confident prospect of success, although in many instances this pessimism proved unfounded. Some 
$19 \%$ of all our patients came to surgery with evidence of preretinal retraction, whether as fixed folds, rolled tear edges, taut opercula, or a rigid vitreous.

We believe that our primary success rate with the need for only one relatively short general anaesthetic must commend this method to more surgeons than those harassed by long operating lists. We contend that this series of 241 cases, accepted with no thought to prognosis, indicates a greater place for the routine use of intravitreal air in retinal detachment surgery.

\section{References}

Chawla, H. B. (1974). Retinal Detachment: The Essentials of Management. Churchill Livingstone: Edinburgh.

Chawla, H. B., and Birchall, C. H. (1973). Intravitreal air in retinal surgery. British Journal of Ophthalmology, 57, 60.

Chisholm, I. A., McClure, E., and Foulds, W. S. (1975). Transactions of the Ophthalmological Societies of the United Kingdom, 95, 167.

Freeman, H. Mackenzie, and Schepens, C. L. (1974). Modern Problems in Ophthalmology, 15, 119.

Rosengren, B. (1938). Acta Ophthalmologica, 16, 177.

Scott, A. S., and Smith, V. H. (1961). British Journal of Ophthalmology, 45, 654 . 